Versatile Approach for the Synthesis of Sequence-Defined

Monodisperse 18- and 20-mer Oligoacrylates

Peer-reviewed author version

HAVEN, Joris; DE NEVE, Jeroen \& JUNKERS, Tanja (2017) Versatile Approach for the Synthesis of Sequence-Defined Monodisperse 18- and 20-mer Oligoacrylates.

In: ACS Macro Letters, 6, p. 743-747.

DOI: 10.1021/acsmacrolett.7b00430

Handle: http://hdl.handle.net/1942/23949 


\title{
Versatile Approach for the Synthesis of Sequence-Defined Monodis- perse 18- and 20-mer Oligoacrylates
}

\author{
Joris J. Haven, ${ }^{\dagger, \ddagger}$ Jeroen A. De Neve, ${ }^{\dagger, \ddagger}$ Tanja Junkers ${ }^{\star, \dagger, ¥}$ \\ ${ }^{\dagger}$ Polymer Reaction Design Group, Institute for Materials Research (imo-imomec), Hasselt University, Campus Die- \\ penbeek, Building D, B-359o Diepenbeek, Belgium \\ $¥$ IMEC division IMOMEC, Wetenschapspark 1, B-359o Diepenbeek, Belgium
}

\begin{abstract}
Linear monodisperse 18- and 20-mer acrylates are obtained via consecutive synthesis of two sequencedefined acrylate 9- and 10-mers, followed by disulfide coupling utilizing reversible addition-fragmentation chain transfer (RAFT) end group chemistry. The sequence-defined oligoacrylates are accessed via consecutive single (SUMI) and multiple (MUMI) unit monomer insertions through RAFT polymerization, using the extensive acrylate monomer library as functional building blocks. Aminolysis of the trithiocarbonate macroRAFT end group and in situ oxidation of the thiols to form a disulfide bridge lead to the formation of 18 - and 20-mer acrylates. In this approach, one or multiple acrylate building blocks can be inserted in each step by chain extension to form a stable carbon-carbon backbone. Isolation of the targeted monodisperse oligomers, from the statistical mixtures obtained at first, is performed by flash column chromatography with high efficiency. It is shown that the SUMI and MUMI strategy, when combined with flash column chromatography separation, is highly efficient and allows to construct monodisperse materials of very considerable length starting from cheap and very versatile building blocks.
\end{abstract}

Biopolymers such as proteins and DNA have highly selective and complex functions, based fundamentally on their primary structure, hence, the specific order of repeating units and functionalities along their backbone. Concomitantly, there is a high need for specialized sustainable materials produced via synthetic routes. Therefore, the synthesis of precision polymers with unprecedented control over the primary monomer sequence is currently widely investigated. ${ }^{1}$ Regulation of the monomer sequence in polymerizations, the so called sequencecontrolled multiblock copolymers having a polydisperse nature, is of high interest to mimic biological materials in their functionality. ${ }^{2}$ Truly following the concept of nature, thus, to synthesize monodisperse materials, so-called sequence-defined polymers, broadens the functionality of these materials further, and opens new perspectives for example for biological and information storage applications. ${ }^{3}$ Iterative and orthogonal growth via nonradical chemical strategies have been reported on this matter. A binary encoded polyphosphate oligomer with a degree of polymerization above 100 was synthesized by iterative Merrifield-based chemistry by Lutz and coworkers. This method being inspired by solid-phase peptide synthesis. ${ }^{4}$ More nonradical iterative approaches up to 10-mers are known in literature. ${ }^{5}$ However, orthogonal and iterative growth strategies can only couple one building block at a time and in many cases require protection/deprotection strategies of the growing oligomer chain which makes it an expensive and time-consuming approach. While some of these methods are able to yield products with high efficiencies, typically monomer building blocks need to be presynthesized, which must be taken into account when assessing the economy of reactions. In here, the synthesis of an acrylate monomer based sequence-defined 18- and 2o-mer are reported via a radical growth strategy in homogeneous liquid phase. It must be noted that the used terminology for all sequences refers to the amount of acrylate monomers incorporated in the sequence. Telechelic monodisperse 9- and 10-mers are produced, which were then coupled via disulfide coupling utilizing RAFT end group chemistry. Counting functional groups per chain, one would need to take polymer end groups into account, yielding 20- and 22-mers as products. However, no clear terminology exists on this matter yet. Counting only the inserted functionalities, in this case acrylate monomers, gives a more conservative number for the sequence length. To to stay on the side of caution this notation has been implemented across this manuscript. 


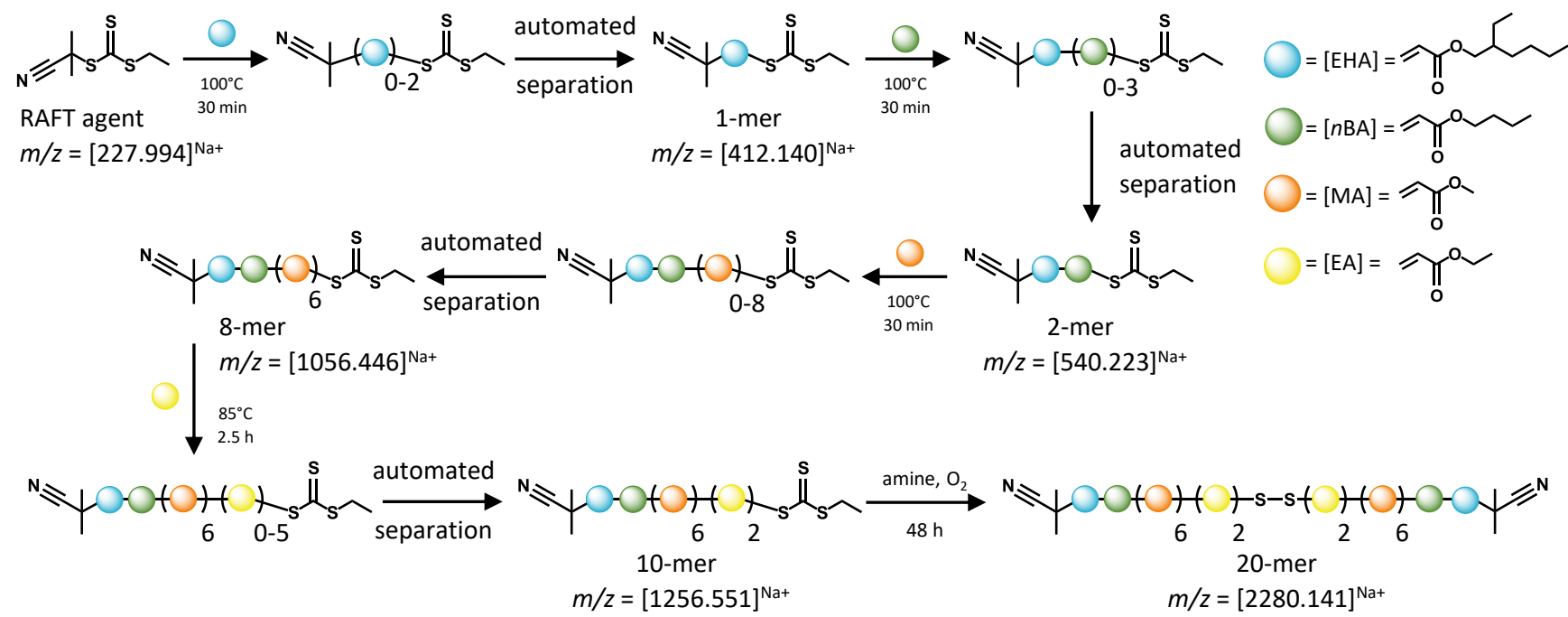

Scheme 1. General reaction scheme for the synthesis of a 20-mer. Four RAFT polymerizations were performed, with purification steps in between via automated separation through flash column chromatography, to obtain a 10-mer. All RAFT polymerizations were thermally initiated at elevated temperatures by 2,2'-azobis(2-methylpropionitrile) (AIBN). The 10-mers were then orthogonally coupled via aminolysis of the RAFT end group followed by in situ oxidation for the formation of a disulfide bridge to yield 20-mers.

Acrylate monomers are known for their high propagation rate thus insertions are fast, typically on the timescale of tens of minutes or faster. Due to the radical process, oligomer growth is not limited to one unit at a time and a rather chemically inert carbon-carbon backbone is grown, making SUMI sequence-defined materials considerably chemically more stable than most counterparts made from iterative strategies, which often require heteroatom incorporation in the main chain. Moreover, chain extension can, after purification, be either mono- or polydisperse and a large versatility of building blocks and functionalities is available. Yet, as a downside, careful purification is required (which is however also the case for many other techniques) and overall product yields are on first glance low due to the separation of products out of statistical mixtures. It must be noted though that isolation of oligomers always results in a library of monodisperse compounds, which can be used for further purposes. In other words, the yield for a single sequence selected to be implemented in a further reaction is rather modest, yet multiple other unique sequence-defined structures are formed as well. This, in combination with the very simple synthesis procedure itself and the broad availability of cheap monomers leads to an overall very economic and efficient synthesis process. In here, oligomers were prepared in a two-step approach. First, a 9- and 10-mer were prepared via precise insertion of a single unit (SUMI) or multiple units (MUMI) of acrylate monomer(s) at a time through reversible addition-fragmentation chain transfer (RAFT) polymerization. Second, the obtained oligomers were orthogonally coupled via aminolysis of the RAFT end group followed by in situ oxidation for the formation of a disulfide bridge. Coupling allows to increase the reachable chain length of the oligomers significantly, but serves also as an ideal tool to demonstrate another advantage of using the well-known controlled radical polymerization (CRP) methods for sequence-defined oligomer synthesis. For CRP, a plethora of click-like coupling techniques have been developed in previous years, which are directly available for the SUMI products without any further modification.

Monodisperse sequences of the targeted oligomer length were isolated via flash column chromatography which is unavoidable due to the statistical polydisperse nature of radical polymerizations as mentioned above. In this study, a range of four different acrylate monomer building blocks was selected, being 2-ethylhexyl acrylate (EHA), n-butyl acrylate ( $n$ BA), methyl acrylate (MA) and ethyl acrylate (EA). Any acrylate monomer that is compatible with RAFT could in principle be chosen for this purpose with an adaptation of the separation protocol. Scheme 1 shows a general reaction scheme for the synthesis of a 20-mer and its assembly in a disulfide coupling reaction. Virtually any other oligomer sequence can likewise be synthesized by varying type and length of each monodisperse building block. In short, to demonstrate the scope of the reaction, single monomer insertions were first carried out using EHA and $n \mathrm{BA}$, respectively. In the next step, a chain length extension of six MA units was performed (see Figure 1). Finally, two further EA units were built into the sequence to yield a 10-mer (see Figure 2). Polydisperse oligomer mixtures were purified by flash column chromatography, a straightforward and scalable technique which Hawker and coworkers recently employed for the separation of homopolymer insertion products. ${ }^{6}$ In previous studies we had used recycling GPC to purify mixtures. ${ }^{7}$ Flash column chromatography has 

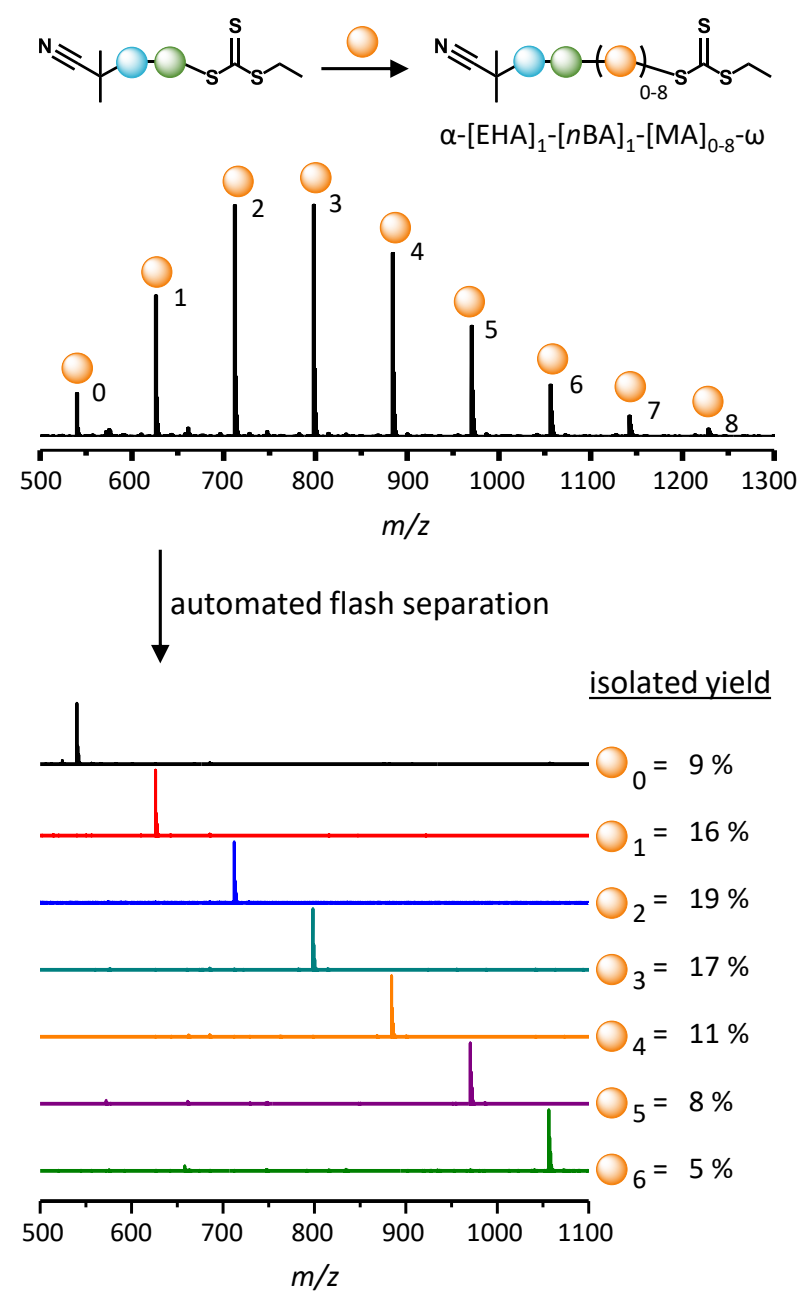

Figure 1. ESI-MS analysis of the oligomer chain extension with six methyl acrylate (MA) units. ESI-MS shows the isolated oligomers after purification via flash column chromatography. The corresponding isolated yields are given at the side.

proven to be not only significantly faster (with separation of the products taking roughly $1 \mathrm{~h}$ ), but also being able to separate higher sequence lengths compared to GPC when polar monomers are employed. Finally, the RAFT $\omega$-end groups of the obtained oligomers were aminolyzed and in situ oxidized for disulfide bridge formation. Via this approach, selected sequences could be coupled to form custom-made precision polymers.

Monodisperse 10- and 20-mers were synthesized according to this procedure. Although all purified products are monodisperse, they still consist out of a mixture of stereoisomers which is inherent to the employed approach. Precise synthesis procedures and product analysis for all steps are described in detail in the supporting information (SI). An indispensable technique for the analysis of oligomer mixtures is hereby electrospray ionization mass spectrometry (ESI-MS). The RAFT control agent 2cyano-2-propyl ethyl trithiocarbonate (CPE-TTC) was synthesized (Supporting Information, section S3.1) to initiate the oligomer sequences and all polymerization steps were thermally initiated by 2,2'-azobis(2methylpropionitrile) (AIBN).

Without going into detail on the exact synthesis procedures here (section $\mathrm{S}_{3}$ in the Supplementary Information), it is important to discuss reaction efficiencies. The first SUMI reaction yielded $1.9 \mathrm{~g}(58 \mathrm{~mol} \%$ isolated yield) of the monodisperse $\alpha$-[EHA $]_{1}-\omega 1$-mer. The following SUMI with $n$-butyl acrylate ( $n \mathrm{BA}$ ) (Scheme 1 ) yielded again a monodisperse macroRAFT $\alpha$ - $[\mathrm{EHA}]_{1}-[\mathrm{nBA}]_{1}-\omega$ mer (540 mg, $45 \mathrm{~mol} \%$ isolated yield). ESI-MS analysis shows $>99 \%$ end group fidelity of the obtained 1- (Figure S4.1) and 2-mers (Figure S4.2). Although purification of the oligomer mixtures is inevitable, reactions can be optimized to obtain maximum yields of the targeted oligomer length as described previously (close to $45 \mathrm{~mol} \%$ was identified to be the maximum achievable crude yield, only for the first insertion higher yields are achievable due to the difference in pre-equilibrium kinetics). ${ }^{8}$ In principle, from here on several SUMIs could be performed as a follow up, each time adding exactly one unit. In the following step, to use the power of RAFT, a multiple unit monomer insertion (MUMI) was performed up to 6 acrylate units at a time. Many sequence-defined natural materials contain homosequences of several units, and a direct insertion of a larger (yet monodisperse) block can be of large advantage. After polymerization of macroRAFT agent $\alpha-[\mathrm{EHA}]_{1}-[n \mathrm{BA}]_{1}-\omega$ with methyl acrylate, $750 \mathrm{mg}$ crude reaction product was loaded on the flash column to isolate $53 \mathrm{mg}$ ( $5 \mathrm{~mol} \%$ isolated yield) of the monodisperse 8 -mer $\alpha$ - $[\mathrm{EHA}]_{1}-[n \mathrm{BA}]_{1}-[\mathrm{MA}]_{6}-\omega$ (all other yields are given in Figure 1). The more units are inserted per step, the lower the overall achievable yield due to the resulting Poisson distribution of products. The polydisperse ESIMS spectrum directly after polymerization of MA shows a chain length distribution from o to 8 insertions of MA. The flash column chromatography system could be easily operated under UV detection due to the high absorption of the trithiocarbonate RAFT end group. ESI-MS of the crude oligomer spectrum shows that higher insertions (> 6 ) are only present in very small amounts. Insertions 7 and 8 were isolated and analyzed by ESI-MS as shown in Figure S4.3. Via this technique almost no product losses are encountered during purification since monomer insertions were baseline separated according to the UV detection signal (Figures S6.1-6.4). More details about the purification procedures are added to the SI (Section S6). Isolated yields shown in Figure 1 are calculated based on the initial amount (mol \%) of macroRAFT agent. For o to 6 insertions of MA $>85 \mathrm{~mol} \%$ of the crude material could be isolated in total, so only minor losses are encountered via this approach taking into account the higher insertion products and little losses due to sample preparation, analysis and purification. Thus, while the individual yield of a single oligomer sequence may be low, the overall yield of all isolated oligomers is rather close to quantitative. 

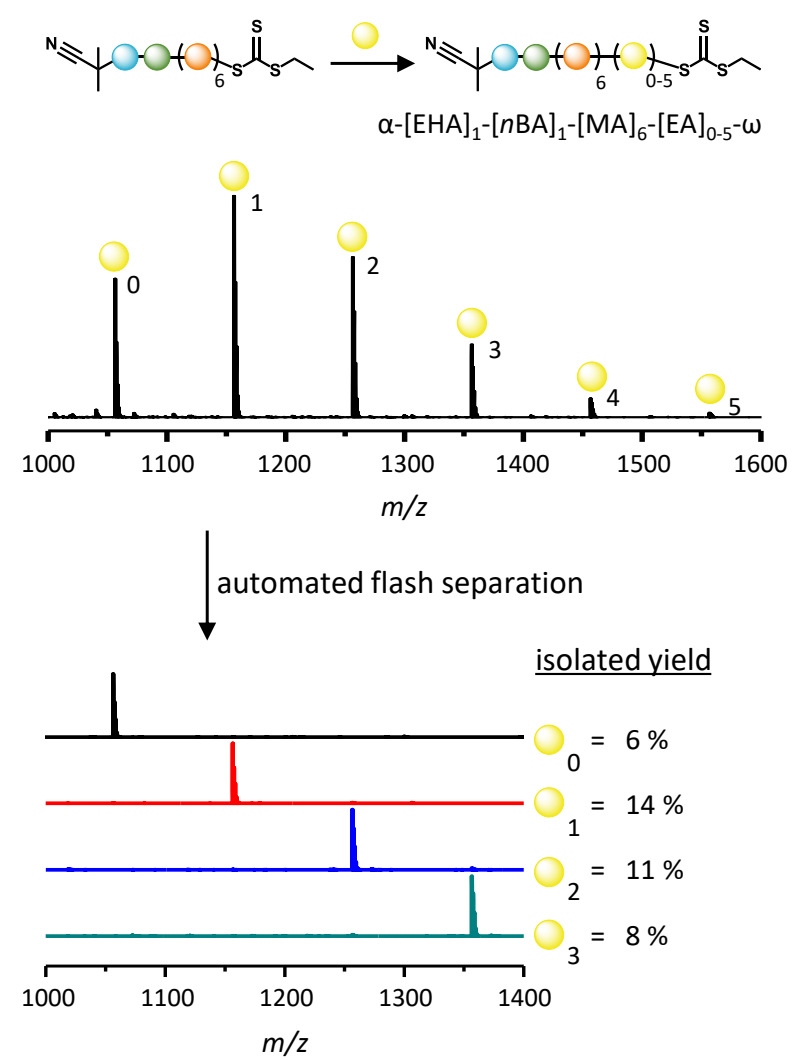

Figure 2. ESI-MS analysis of the oligomer chain extension with three ethyl acrylate (EA) units. ESI-MS spectra show the isolated oligomers after purification via flash column chromatography. The corresponding yields are given at the side.

Figure 2 shows the final RAFT polymerization step where the sequence was chain extended with ethyl acrylate as a fourth monomer building block to obtain $\alpha$ $[\mathrm{EHA}]_{1}-[n \mathrm{BA}]_{1}-[\mathrm{MA}]_{6}-[\mathrm{EA}]_{\mathrm{x}}-\omega$. One, two and three insertions of EA could be isolated as monodisperse sequences yielding $8 \mathrm{mg} 9$-mers ( $14 \mathrm{~mol} \mathrm{\%}$ isolated yield), $7 \mathrm{mg} 10-$ mers (11 mol \% isolated yield) and $6 \mathrm{mg} 11$-mers ( $8 \mathrm{~mol} \%$ isolated yield). It can be observed that more losses are encountered which can be explained by mixed insertion fractions obtained after column chromatography and the beginning loss of separation resolution with increasing chain lengths (Figure S6.5). Overall yields obtained for the 9-, 10- and 11-mers, after four chain extensions, are in the range of a few percent. However, the synthesis of the targeted oligomer sequences is still economically feasible since starting materials are cheap and commercially available. Moreover, purification of the polydisperse oligomer mixtures via flash column chromatography is fast, which limits eluent consumption. More precisely, purification of the final 10-mer sequence was performed in less than 1 hour (Figure S6.5). Overall, synthesis is fast and sequences can be built up rapidly without the use of solid phase synthesis strategies, even if product amounts are low when compared to Merrifield synthesis approaches.

As widely investigated in literature, so-called midchain radicals can be formed via backbiting during
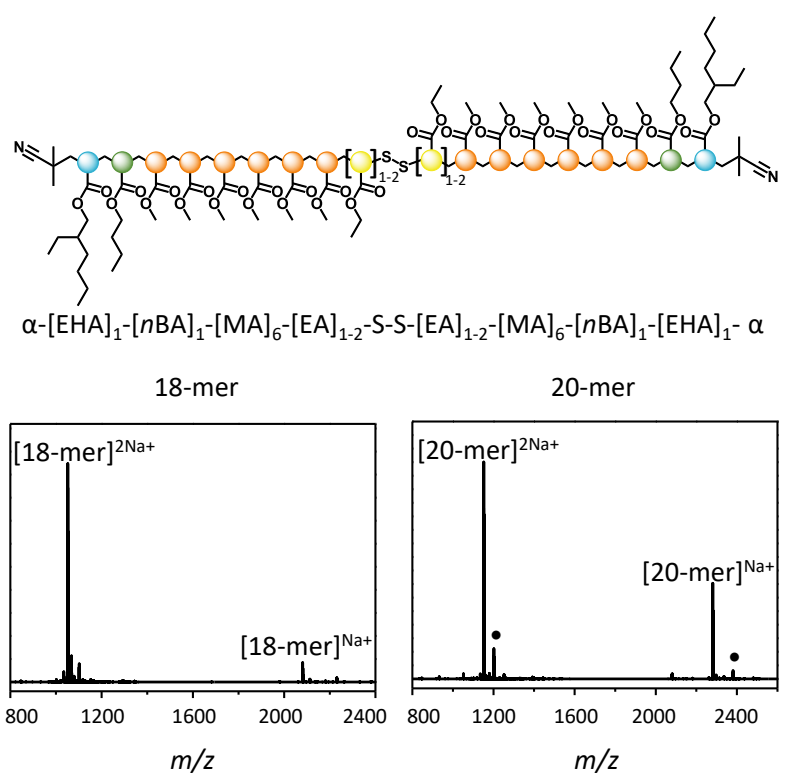

Figure 3. Representation of the synthesized 18- and 20-mer. Monodisperse oligomers were obtained as shown by ESI-MS. The marked signals are assigned to a single and double sodium charged 21 -mer $(<10 \%)$ due to coupling of a 10- and 11mer trace left from the starting material.

acrylate propagation due to the formation of a more stable tertiary radical. These radicals can undergo propagation and cause branched polymer structures, a phenomenon that is well known. ${ }^{9}$ Obviously, branching would disturb the pristine sequence desired. While ESI-MS shows the monodisperse nature of the isolated oligomers, it does not give any information on the exact macromolecular architecture since structural branched isomers have exactly the same mass-to-charge signal in ESI-MS (isobaric structures). To exclude branching and to proof a linear growth of the oligomers without defects in the structure, ${ }^{13} \mathrm{C}$ and attached proton test (APT) carbon NMR spectra where taken, confirming the complete absence of any branched structure in the final products. It can be speculated that branched structures are associated with considerably different polarities and are hence separated out in the chromatography process, see details in the SI (Section $\left.\mathrm{S}_{5}\right)$.

Figure 3 demonstrates the disulfide coupling toward the final 18- and 20-mers as an example of how RAFT SUMI reactions can be combined with postoligomerization reactions. RAFT chemistry allows for numerous click-like reactions, ranging from azide-alkyne coupling over Diels-Alder cycloadditions to thiol-ene chemistry. Disulfide coupling was chosen herein to demonstrate the synthetic potential as disulfide bridges are a common motif in biomolecule conjugation, and because we see the future of the SUMI-made sequencedefined materials in this field. To carry out the coupling, the trithiocarbonate RAFT end group is reduced by aminolysis, a fast and quantitative reaction. Disulfide bridges form under oxidative conditions of the residual thiol end 
groups in air. The coupling products were purified by manual silica column chromatography (to remove ethane-thiol coupling side products) and analyzed by ESIMS. The mass spectra (Figure 3) exhibit a double and single sodium charged species of the 18 - (Figure $3 \mathrm{~A}$, 2057.05 $\mathrm{g} \cdot \mathrm{mol}^{-1}$ ) and 20-mer (Figure 3B, $2257.15 \mathrm{~g} \cdot \mathrm{mol}^{-1}$ ). Some small side products can also be identified in the 20mer product, these can be assigned to single- and double sodium charged 21 -mers $(<10 \%)$ due to coupling of a $10-$ and 11-mer trace left from the starting material. These side products occur only at very low abundancy.

In conclusion, an effective and flexible route toward the synthesis of sequence-defined oligomers was established. Using flash column chromatography has significantly advanced the reachable sequence length of the materials, now unfolding the full potential of using RAFT for the synthesis of sequence-defined materials. The 18and 20-mers with a precisely defined monomer order could be synthesized, allowing for single monomer insertions or for insertion of several monomer units at a time with a large tolerance toward functional groups. Separation of the various insertion products via flash column chromatography resulted in the fast $(<1 \mathrm{~h})$ and elegant (baseline) purification of reaction mixtures. The materials described herein mark an unprecedented length for sequence-defined materials made from radical reactions, and are highly competitive with iterative synthesis strategies. Individual yields of the different sequences are relatively low, yet in each step a library of compounds is obtained. This plethora of sequences, each with a varying structure, can be of significant interest to study the property differences between them. Furthermore, a simple and fast synthesis process is presented. All monomers are cheap and commercially available and synthesis time is minimal. Also purifications typically do not consume much time (typically 60 min per separation), allowing for comparatively fast oligomer separation. ESI-MS and APT ${ }^{13} \mathrm{C}$ NMR confirmed the high structural integrity of the products. Furthermore, the successful coupling of two monodisperse blocks of a specified length has been demonstrated, resulting in 18- and 20-mers. This straightforward synthesis of such sequence-defined block copolymers truly pushes the boundaries of sequence-controlled materials and paves the way to numerous innovations in the future in the field of material chemistry with the possibility to create new, highly-customizable materials.

\section{ASSOCIATED CONTENT}

\section{Supporting Information}

The supporting information is available free of charge on the ACS Publications website at DOI: 10.1021/acsmacrolett.7boo43o. Procedures and additional characterization data (PDF).

\section{AUTHOR INFORMATION}

\section{Corresponding Author}

*E-mail: tanja.junkers@uhasselt.be

\section{Author Contributions}

†These authors contributed equally.

\section{Notes}

The authors declare no competing financial interest.

\section{ACKNOWLEDGMENT}

The authors acknowledge funding via the Fonds Wetenschappelijk Onderzoek (FWO) and the Bijzonder Onderzoeksfonds (BOF) of Hasselt University.

\section{REFERENCES}

(1) (a) Lutz, J.-F. Sequence-controlled polymerizations: the next Holy Grail in polymer science? Polym. Chem 2010, 1, 55-62. (b) Lutz, J.-F.; Ouchi, M.; Liu, D.R.; Sawamoto, M. Sequence-Controlled Polymers. Science 2013, 341, 1238149. (c) Lutz, J.-F. Coding Macromolecules: Inputting Information in Polymers Using Monomer-Based Alphabets. Macromolecules 2015, 48, 4759-4767.

(2) (a) Guillaume, G.; Maschmeyer, T.; Zetterlund, P.B.; Perrier, S. Rapid and quantitative one-pot synthesis of sequence-controlled polymers by radical polymerization. Nat. Commun. 2013, 4, 2505. (b) Boyer, C.; Soeriyadi, A.H.; Zetterlund, P.B.; Whittaker, M.R. Synthesis of Complex Multiblock Copolymers via a Simple Iterative $\mathrm{Cu}(\mathrm{o})$ Mediated Radical Polymerization Approach. Macromolecules 2011, 44, 8028-8033. (c) Engelis, N.G.; Anastasaki, A.; Shegiwal, A.; Whittaker, M.R.; Nurumbetov, G.; Davis, T.P.; Truong, Nikolaou, V.; N.P.; Haddleton, D.M. Sequence-controlled methacrylic multiblock copolymers via sulfur-free RAFT emulsion polymerization. Nat. Chem. 2016, 10.1038/nchem.2634. (d) Chuang, Y.; Ethirajan, A.; Junkers, T. Photoinduced Sequence-Controlled Copper-Mediated Polymerization: Synthesis of Decablock Copolymers. ACS Macro Lett. 2014, 3, 732-737. (e) Anastasaki, A.; Nikolaou, V.; Nurumbetov, G.; Wilson, P.; Kempe, K.; Quinn, J.F.; Davis, T.P.; Whittaker, M.R.; Haddleton, D.M. $\mathrm{Cu}(\mathrm{o})$-Mediated Living Radical Polymerization: A Versatile Tool for Materials Synthesis. Chem. Rev. 2016, 116, 835-877. (f) Nurumbetov, G.; Engelis, N.; Godfrey, J.; Hand, R.; Anastasaki, A.; Simula, A.; Nikolaou, V.; Haddleton, D.M. Methacrylic block copolymers by sulfur free RAFT (SF RAFT) free radical emulsion polymerization. Polym. Chem., 2017, 8, 1084-1094. (g) Haven, J.J.; GuerreroSanchez, C.; Keddie, D.J.; Moad, G. Rapid and Systematic Access to Quasi-Diblock Copolymer Libraries Covering a Comprehensive Composition Range by Sequential RAFT Polymerization in an Automated Synthesizer. Macromol. Rapid Commun. 2o14, 35, 492-497. (h) Gody, G.; Barbey, R.; Danial, M.; Perrier, S. Ultrafast RAFT polymerization: multiblock copolymers within minutes. Polym. Chem., 2015, 6, 1502-1511. (i) Haven, J.J.; Guerrero-Sanchez, C.; Keddie, D.J.; Moad, G.; Thang, S.H.; Schubert, U.S. One pot synthesis of higher order quasi-block copolymer libraries via sequential RAFT polymerization in an automated synthesizer. Polym. Chem., 2014, 5, 5236-5246.

(3) (a) Lutz, J.-F.; Lehn, J.-M.; Meijer, E.W.; Matyjaszewsk, K. From precision polymers to complex materials and systems. Nat. Rev. Mat. 2016, 1, 16024. (b) Roy, R.K.; Meszynska, A.; Laure, C.; Charles, L.; Verchin, C.; Lutz, J.-F. Design and synthesis of digitally encoded polymers that can be decoded and erased. Nat. Commun. 2015, 6, 7232. (c) Houshyar, S.; Keddie, D.J.; Moad, G.; Mulder, R.J.; Saubern, S.; Tsanaktsidis, J. The scope for synthesis of macro-RAFT agents by sequential insertion of single monomer units. Polym. Chem. 2012, 3, 1879-1889. (d) Moad, G.; Guerrero-Sanchez, C.; Haven, J. J.; Keddie, D. J.; Postma, A.; Rizzardo, E.; Thang, S. H. In Sequence-Controlled Polymers: Synthesis, Self-Assembly, and Properties; American Chemical Society: Washington, D.C., 2o14; Vol. 1170, pp 133-147.

(4) (a) Ouahabi, A.A.; Kotera, M.; Charles, L.; Lutz, J.F. Synthesis of Monodisperse Sequence-Coded Polymers with Chain Lengths above DP10o. ACS Macro Lett. 2015, 4, 1077-1080. (b) Caruthers, M. H. Gene synthesis machines: DNA chemistry and its uses. Science 1985, 230, 281-285. 
(5) (a) Susanne, S.C.; Deniz, Z.; Katharina, W.S.; Meier, M.A.R. A Scalable and High-Yield Strategy for the Synthesis of SequenceDefined Macromolecules. Angew. Chem., Int. Ed. 2016, 55, $2105-1207$. (b) Martens, S.; Van den Begin, J.; Madder, A.; Du Prez, F.E.; Espeel, P. Automated Synthesis of Monodisperse Oligomers, Featuring Sequence Control and Tailored Functionalization. J. Am. Chem. Soc. 2016, 138, 14182-14185. (c) Zydziak, N.; Feist, F.; Huber, B.; Meuller, J.O.; Barner-Kowollik, C. Photo-induced sequence defined macromolecules via hetero bifunctional synthons. Chem. Commun. 2015, 51, 1799-1802.

(6) Lawrence, J.; Lee, S.-H.; Abdilla, A.; Nothling, M.D.; Ren, J.M.; Knight, A.S.; Fleischmann, C.; Li, Y.; Abrams, A.S.; Schmidt, B.V. K. J.; Hawker, M.C.; Connal, L.A.; McGrath, A.J.; Clark, P.G.; Gutekunst, W.R.; Hawker, C.J. A Versatile and Scalable Strategy to Discrete Oligomers. J. Am. Chem. Soc. 2016, 138, 6306-6310.

(7) (a) Vandenbergh, J.; Reekmans, G.; Adriaensens, P.; Junkers, T. Synthesis of sequence controlled acrylate oligomers via consecutive RAFT monomer additions. Chem. Commun. 2013, 49, 10358-1036o. (b) Vandenbergh, J.; Reekmans, G.; Adriaensens, P.; Junkers, T. Synthesis of sequence-defined acrylate oligomers via photo-induced copper-mediated radical monomer insertions. Chem. Sci. 2015, 6, 5753-5761.

(8) (a) Haven, J.J.; Vandenbergh, J.; Kurita, R.; Gruber, J.; Junkers, T. Efficiency assessment of single unit monomer insertion reactions for monomer sequence control: kinetic simulations and experimental observations. Polym. Chem. 2015, 6, 5752-5765. (b) Haven, J.J.; Vandenberg, J.; Junker, T. Watching polymers grow: real time monitoring of polymerizations via an on-line ESI-MS/microreactor coupling. Chem. Commun. 2015, 51, 4611-4614.

(9) (a) Wenn, B.; Reekmans, G.; Adriaensens, P.; Junkers, T. Photoinduced Acrylate Polymerization: Unexpected Reduction in Chain Branching. Macromol. Rapid Commun. 2015, 36, 14791485. (b) Gaborieau, M.; Koo, S.P.S.; Castignolles, P.; Junkers, T.; Barner-Kowollik, C. Reducing the Degree of Branching in Polyacrylates via Mid-Chain Radical Patching: A Quantitative Melt-State NMR Study. Macromolecules 2o1o, 51, 5492-5495. 
Linear monodisperse 18- and 20-mer oligoacrylates are obtained via consecutive synthesis of two sequence-defined acrylate 9- and 10-mers followed by disulfide coupling. The sequence defined oligoacrylates are accessed via single unit (SUMI) and multiple (MUMI) unit monomer insertions through reversible addition-fragmentation chain transfer (RAFT) polymerization, using the extensive acrylate monomer library as functional building blocks.

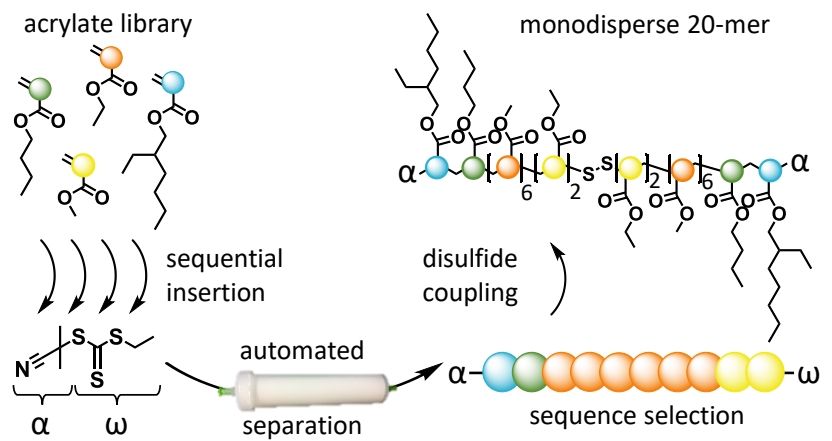

